\title{
A!
}

This is an electronic reprint of the original article.

This reprint may differ from the original in pagination and typographic detail.

Dolejsova, Marketa; Wilde, Danielle; Altarriba Bertran, Ferran; Davis, Hilary

\section{Disrupting (More-than-) Human-Food Interaction}

\section{Published in:}

DIS 2020 - Proceedings of the 2020 ACM Designing Interactive Systems Conference

DOI:

$10.1145 / 3357236.3395437$

Published: 03/07/2020

Document Version

Peer reviewed version

Please cite the original version:

Dolejsova, M., Wilde, D., Altarriba Bertran, F., \& Davis, H. (2020). Disrupting (More-than-) Human-Food Interaction: Experimental Design, Tangibles and Food-Tech Futures. In DIS 2020 - Proceedings of the 2020 ACM Designing Interactive Systems Conference (pp. 993-1004). ACM.

https://doi.org/10.1145/3357236.3395437

This material is protected by copyright and other intellectual property rights, and duplication or sale of all or part of any of the repository collections is not permitted, except that material may be duplicated by you for your research use or educational purposes in electronic or print form. You must obtain permission for any other use. Electronic or print copies may not be offered, whether for sale or otherwise to anyone who is not an authorised user. 


\section{Disrupting (More-than-) Human-Food Interaction: Experimental Design, Tangibles and Food-Tech Futures}

\author{
Markéta Dolejšová* \\ Aalto University \\ Espoo, FI \\ marketa.dolejsova@ \\ aalto.fi
}

\author{
Danielle Wilde* \\ University of Southern \\ Denmark \\ Kolding, DK \\ wilde@sdu.dk
}

\author{
Ferran Altarriba \\ Bertran \\ UC Santa Cruz \\ Santa Cruz, CA, USA \\ faltarri@ucsc.edu
}

\author{
Hilary Davis \\ Swinburne University of \\ Technology \\ Melbourne, AU \\ hdavis@swin.edu.au
}

\begin{abstract}
Digital technology has become a frequent companion of daily food practices, shaping the ways we produce, consume, and interact with food. Smart kitchenware, diet tracking apps, and other techno-solutions carry promise for healthy and sustainable food futures but are often problematic in their impact on food cultures. We conducted four Human-Food Interaction (HFI) workshops to reflect on and anticipate food-tech issues, using experimental food design co-creation as our primary method. At the workshops, food and food practices served as the central research theme and accessible starting point to engage stakeholders and explore values, desires, and imaginaries associated with food-tech. Drawing on these explorations, we discuss diverse roles that experimental design cocreation, performed with and around food, can play in supporting critical, interdisciplinary HFI inquiries. Our findings will appeal to design researchers interested in food as a research theme or as a tangible (and compostable!) design material affording diverse co-creative engagements.
\end{abstract}

\section{AUTHOR KEYWORDS}

Human-Food Interaction, HFI, food futures, experimental food design, embodied design, tangibles, compostables

\section{CCS CONCEPTS}

- Human-centered computing Interaction design

\section{INTRODUCTION}

Human-food practices are key drivers of personal and planetary health and have the potential to nurture both. However, current modes of food production and consumption are causing ill health and amplifying climate change [38]. A burgeoning realm of food-tech entrepreneurs and venture capitalists propose solutions for healthier, more sustainable and more efficient food

\footnotetext{
Permission to make digital or hard copies of all or part of this work for personal or classroom use is granted without fee provided that copies are not made or distributed for profit or commercial advantage and that copies bear this notice and the full citation on the first page. Copyrights for components of this work owned by others than ACM must be honored. Abstracting with credit is permitted. To copy otherwise, or republish, to post on servers or to redistribute to lists, requires prior specific permission and/or a fee. Request permissions from Permissions@acm.org. DIS '20, July 6-10, 2020, Eindhoven, Netherlands

(C) 2020 Association for Computing Machinery.

ACM ISBN 978-1-4503-6974-9/20/07 ...\$15.00

https://doi.org/10.1145/3357236.3395437
}

practices - from smart kitchenware and diet personalization services to food sharing apps and digital farming platforms. Yet, such food-tech solutions offer uncertain food futures and are often problematic in their impacts on food cultures and practices: they cause negative changes to social food traditions, deepen socio-economic inequalities on global food markets, jeopardise consumers' privacy, and more $[11,14]$.

Risks and opportunities of food-tech innovation are at the center of Human-Food Interaction (HFI) - a growing research area that traverses multiple disciplines and draws on diverse approaches to bring focus to the interplay between humans, food, and technology [3]. Motivated by a shared belief that HFI has an important role in supporting positive, socially and ecologically sustainable food futures, we formed an HFI community network Feeding Food Futures (FFF) [17]. The FFF network investigates foodtech practices, issues, and opportunities through experimental food design co-creation. Within the network, we organise design research workshops to support critical and creative HFI engagements with the social, cultural, environmental and political implications of augmenting food practices with technology. In these workshops, food and food practices serve as a primary research theme, design material, and accessible starting point from which to explore and articulate values, concerns, desires, and imaginaries associated with food and food-tech futures.

Food has a number of qualities, important for design research. Food practices are relatable events in everyday life that occur at the scale of the body - the scale at which people operate, think and easily imagine. Food is socioculturally potent, materially-rich and aesthetically-charged. Unlike other design materials, by definition, food is edible. It is also perishable and compostable, and may be left to decompose during or after a research experiment. Food thus extends to more-than-human life as a key concern in creating sustainable living systems. Through all of these qualities, designing co-creative experiments with food and food practices affords accessible, imaginative and sustainable material interactions within and beyond the practice of research.

* Dolejšová \& Wilde are co-first authors of this paper 
In this paper, we focus on four food-oriented workshops held at DIS, CHI and CHI Play $[6,11,14,32]$. We introduce the workshop backgrounds and participants, and follow with a detailed overview of six distinct areas of experimental food design methods and approaches deployed at the workshops. We unpack the food-tech activities, detail the debates and reflections that they provoked at the workshops, and outline diverse roles that experimental design co-creation - performed with and around food - can play in supporting critical, interdisciplinary HFI inquiry. Drawing on our workshop findings, we propose a set of recommendations for HFI design and research; including the need to open spaces for hands-on experimentation and learning, nurture social and environmental sustainability, preserve cultural traditions, and embrace more-than-human perspectives across the food landscape. We also unfold the opportunities that designing with sensorially-stimulating and compostable qualities of food materials affords for embodied and experimental design that uses tangibles $[9,30,37]$. Our findings will thus appeal to design research practitioners interested in food as a research theme or as a design material that enables diverse co-creative and sustainable engagements.

\section{HFI WORKSHOPS}

Our discussion stems from four day-long conference workshops: Designing Recipes for Digital Food Futures (CHI'18, Montreal), Handmaking Food Ideals: Crafting the Design of Future Food-Related Technologies (DIS'18, Hong Kong), Crafting and Tasting Issues in Everyday Human-Food Interactions (DIS'19, San Diego), and Chasing Play Potentials in Food Culture (CHI Play'19, Barcelona) $[6,11,14,32]$. The workshops brought together design researchers, artists, practitioners, and thinkers keen on exploring the diverse roles of technology innovation in everyday food practices. Participants were from varying backgrounds and shared diverse design and research approaches, ranging from ethnographic engagements in food communities to DIY biohacking and digital food fabrication. These divergent approaches to HFI afforded polarised, friendly debates about the desired role of digital technology in food cultures and the contributions we might expect from HFI as a research field.

Our debates were provoked and extended through cocreative experimental food design activities including 1) experimental cooking \& food crafting, 2) situated food-play design, 3) critical food futuring \& speculations, 4) reflecting through food-related boundary objects, 5) local food foraging and tasting, and 6) HFI mapping and zinemaking. We deployed a variety of bespoke food design props and kits, such as Food Tarot cards [28] to provoke future food imaginaries, the Fun'o'meter to assess playful food traditions (see below), and the HFI Lit Review App [3] to search and categorise the corpus of related research publications. Supported by these tools, the workshop activities provoked critical and creative engagements of diverse HFI stakeholders in collective sense-making of

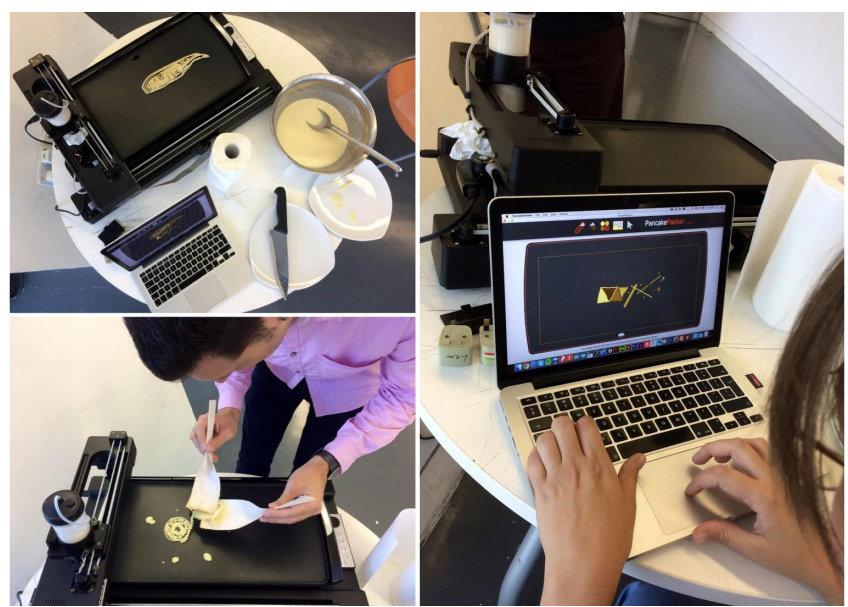

Figure 1: Automated pancake-making with PancakeBot.

food-tech practices and issues. Following, we present our workshop methods and approaches categorised into six primary areas, and highlight the critical debates and discussions that they provoked.

\section{METHODS, APPROACHES, DEBATES 1. Experimental cooking \& food crafting}

At DIS'18, we aimed to explore and compare the potential for creative human-food interactions afforded by i) novel technology-enabled and ii) traditional food making tools and techniques. We experimented with two distinct approaches to making pancakes: the first leverages automated technology using the PancakeBot [7] - a machine that prints pancakes based on drawings made in custom software; the second leverages traditional methods for making pancakes using stove and frypan. Our objective was to enable materially engaged, sensorially diverse, embodied reflection on food-tech innovation. Our expectations were modest. Yet, the contrast between the two approaches was dramatic. With PancakeBot, the cooks' engagement was heavily mediated and largely constrained to preparation: they imagined, then drew a shape on rudimentary software, positioned a plunger containing the batter, and hit a virtual button to begin the 'fully automated' pancake-making process. They then monitored the performance of the robotic chef, before retrieving their pancakes (Figure 1).

In stark contrast, the frypan method required hands-on, embodied, improvisational engagement with food materials and cooking equipment throughout. Significantly, the frypan cooks often overlooked the fact that their activities were mediated through tools. Rather, they seemed guided, far more powerfully, by their senses. With PancakeBot, the machine's affordances radically constrained how we engaged with the organoleptic qualities of the food - i.e., the taste, color, odor, texture and other sensual and material qualities. We found that the machine's functionality was largely 'hands-off' and oriented our attention towards the visual aesthetics of the pancakes. Excited by what the PancakeBot technology seemed to promise, we adapted our 
batter recipe to achieve better flow through the machine's extrusion mechanisms: anticipating more sophisticated visual results; disregarding any impact of this adjustment on taste. Curiously, our experiences with the PancakeBot later influenced our activities when cooking with the frypan: having experienced the PancakeBot's capacity to produce intricate shapes, those of us who used traditional frypan methods also began to focus our attention towards these aesthetic qualities, prioritising the shape of our pancakes over their taste or texture.

This shift to privilege visual aesthetics over other organoleptic qualities demonstrates a key risk of inserting digital technology into material practice. Depending on how technologies are designed, they may cause users to overlook important sensual qualities that sit at the core of material cultures. We determined that, to add value, digital augmentations should consider the complex organoleptic qualities of food - i.e. they should engage cooks' senses, and privilege the complex, socio-sensual nature of food and eating over technological capabilities. We further maintained that digital cooking technologies should include mechanisms to enable human intervention in emergent, improvisational and embodied ways that go beyond instructing a machine to prepare food. Many smart cooking technologies - e.g. June, the autonomous oven [22] emphasize full automation of the cooking process, transferring creativity and responsibility from a human cook to a smart machine. At the DIS'18 workshop, we discussed how such smart cooking diminishes the sensory involvement in meal preparation in favor of comfort and convenience, inviting human users to "sit back and relax" while the technology "does the job" [22]. We raised questions about what the human's "job" should actually be when preparing food, and foregrounded the need for HFI designs to maintain a careful balance between technological efficiency and user agency - the ability to be actively and creatively involved in food practices.

In another DIS'18 experiment, we made pasta from scratch (Figure 2). The purpose was to observe the role of sensorial engagement in food preparation. At different stages in the dough-making process, novice pasta-makers looked for guidance about whether their dough had achieved the qualities necessary for good pasta. Our co-author, pastaexpert assessed the dough by feeling it with her hands and the four, novice pasta-makers all reached to feel the dough in question, to gain an embodied understanding of her evaluations. This process emphasises the importance of evaluating the full range of organoleptic qualities when preparing food. Visuals alone may not communicate what the cook needs to know.

Key takeaways: HFI designs should support creative and experimental food experiences, attending to the full organoleptic experience of food, rather than deliver quickfix solutions aimed at consumer convenience.

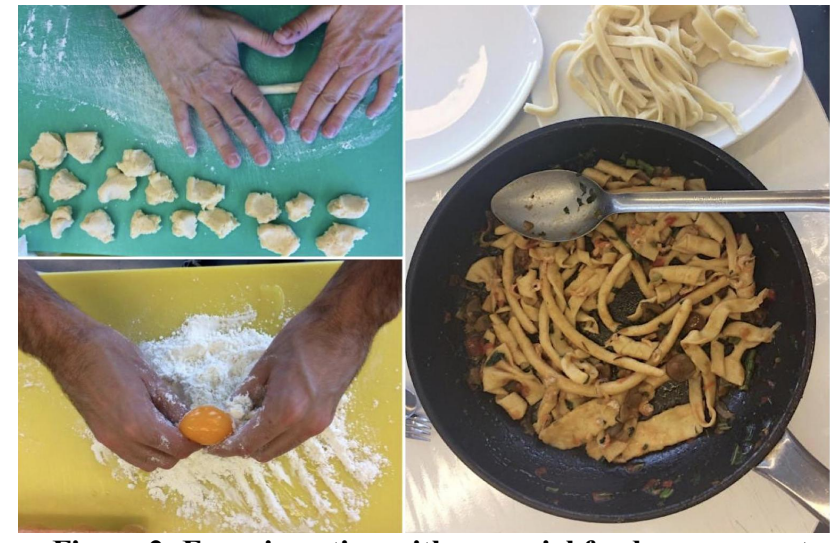

Figure 2: Experimenting with sensorial food engagements.

\section{Situated food-play design}

At CHI Play'19, we explored how food culture and traditions might serve as inspiration to design playful HFI technology that better responds to people's social, cultural and emotional needs. Our goal was to collect culturally diverse playful food traditions to uncover and make design use of their underlying play potentials: contextuallygrounded experiential qualities and interaction mechanisms that promote playful social engagement [4]. Participants contributed with 'playful food traditions' from their personal cultures, which we collected in advance. We worked with an open definition of what a 'playful food tradition' might be to afford diversity in the proposals. An example is Pimientos del Padrón, a Spanish "tapa", or snack, of grilled small green peppers with a unique trait: only some are spicy. This characteristic creates an opportunity for social play: a "Russian roulette" style thrill of not knowing if the pepper you are putting in your mouth is spicy, combines with the excitement of seeing others eating a spicy one. At the workshop, we used a custom toolkit of five experimental design research tools to metaphorically and - where possible - literally play with and thereby reflect on the proposed food traditions. These are:

- Playful Lenses: a check-box document including theoretical concepts of play and HFI. Participants use it to analyze playful food traditions.

- Fun'o'meter: a tangible conversation tool - a box containing diverse data visualizations with blank labels. Participants have to come up with their own criteria for assessing the playful side of traditions.

- Tweak the Tradition: a card deck enclosed in a burger box invites participants to analyze food traditions through disruption. The cards suggest 'what if' scenarios that help players imagine ways of modifying the traditions.

- Blooper Potentials: a movie clapperboard and a prompt to record enactments of potential bloopers with the traditions.

- Play a Role: a box of props and character sheets to enable participants to dress up as clichéed characters (e.g. a grumpy person, a silly person...) and enact traditions. 


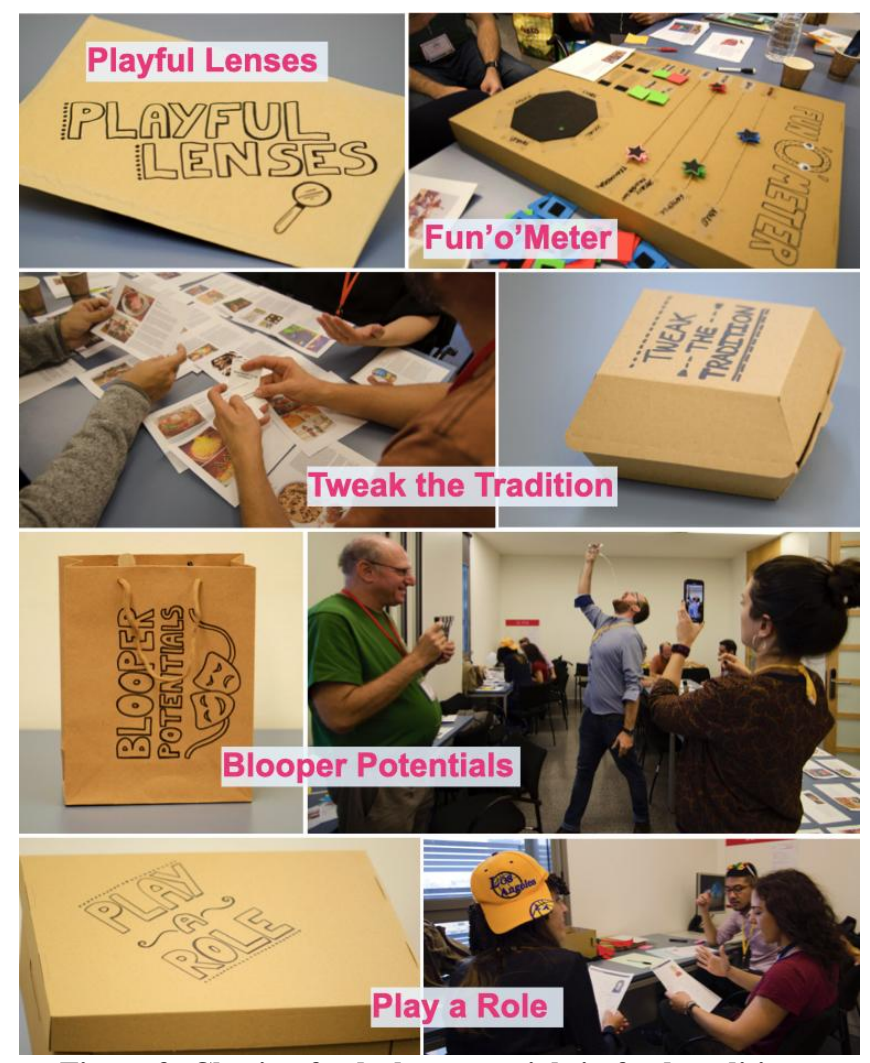

Figure 3: Chasing food-play potentials in food traditions.

These tools were inspired by design research approaches ranging from analytical strategies (e.g. using theoretical frameworks [5]) to embodied design research methods (e.g. embodied sketching [25]). Working with the tools in small groups, we explored the proposed traditions, identified play potentials, and documented our findings on post-its (Figure $3)$. All groups then shared their play potentials and we used thematic analysis to uncover recurrent patterns and mechanisms. Building on our findings we regrouped and rapid-prototyped food-play experiences, artifacts, and technologies inspired by the identified play potentials. We worked with diverse lo-fi prototyping materials including food ingredients, utensils, and Play-d'Oh as well as rapid prototyping technologies such as a Makey Makey board.

Figure 4 illustrates a food-play prototyping process: this group was inspired by El Porró, a Catalan food tradition that involves people drinking from a special vessel that streams wine out of a small nozzle. The play potential is the estrangement produced by the vessel: drinking with it is a challenge that often results in stained shirts. Workshop participants analyzed El Porró, using the Blooper Potentials tool, and prototyped a lo-fi mechatronic food vessel that adds challenge to eating by changing shape as people eat.

The embodied food prototyping inspired by the food-play design toolkit enabled us to reclaim experiences with food that are grounded in cultural practices. It focused our attention on local, traditional food knowledge rather than

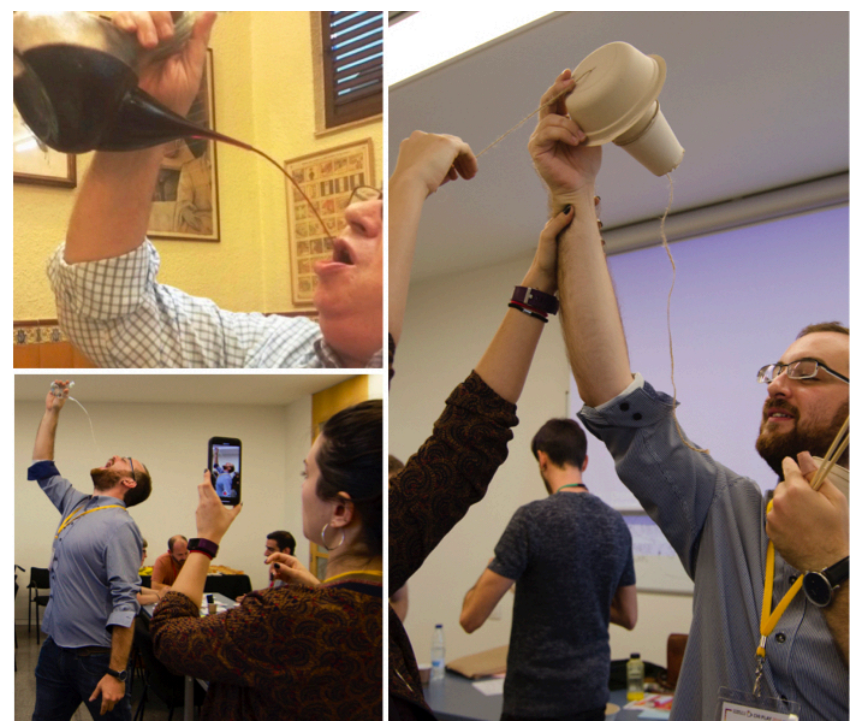

Figure 4: Sequence of a play-food potential chased within a Catalan tradition El Porró.

utilitarian agendas around food. This shift challenges the norm in contemporary HFI research, which often focuses on the efficiency of food practices [3]. While we recognise potential value in such priorities, too much focus on efficiency and optimization of human-food interactions risks compromising important socio-cultural elements of food practices. Our experiments demonstrate that playful, experimental approaches to food-tech design can assist HFI authors to transcend the often uncritical hype of food-tech innovation to imagine socio-cultural food experiences that are sustainable, contextually meaningful, and fun.

Key takeaways: HFI designs should nurture food experiences that are socially engaging, culturally aware, and playful. Designers can take inspiration from local food knowledge and cultural traditions.

\section{Critical food futuring and speculations}

At CHI'18 and DIS'19, we experimented with speculative design approaches to support critical thinking around nearfuture food technologies [20]. We deployed our Food Tarot tool [28]: a card deck presenting 22 imagined diet tribes, illustrating emerging food-tech practices and sociotechnical issues (Figure 5). Working in small groups, we used the deck to envision plausible food-tech futures: each group chose a card and associated it with an HFI project presented during the morning workshop introductions. From this material, we crafted (drew, wrote, imagined) future scenarios. We provide two exemplars.

One group paired the Datavores card (which describes Quantified Self dieters tracking their food practices) with an HFI project that uses digital food-photo journaling to track a person's eating habits, share the habits with their family, and enable remote group support [27]. From this material, they developed a scenario called Total Food Control. It describes a dim future where citizens' food practices and metabolic processes are monitored and evaluated by the 


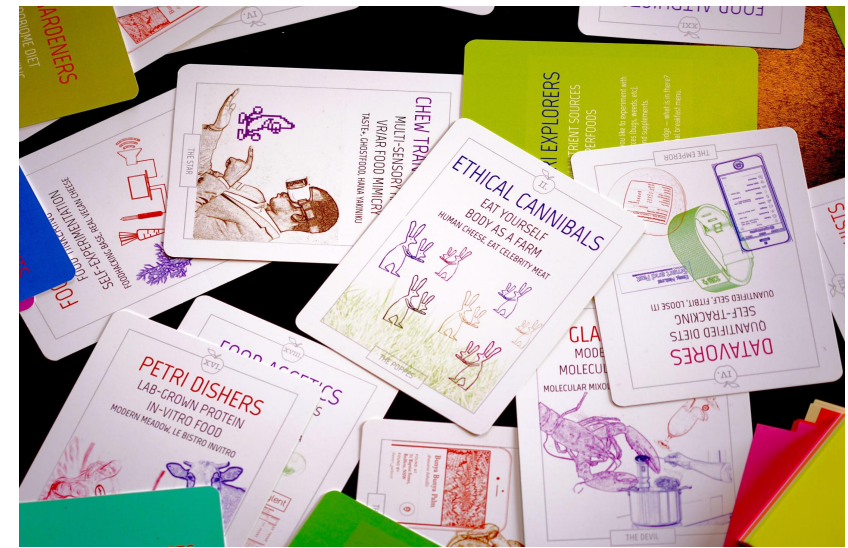

Figure 5: Food Tarot cards and future diet tribes. e.g. Petri Dishers only eat lab-grown meat, Datavores are Quantified Self dieters, Ethical Cannibals tweak their microbiome to grow food in and on themselves. Full deck: [28].

Ministry of Quantified Health. The Ministry designs meals for all citizens according to their data records, ignoring personal preferences. The objective is to keep everyone fit, efficient, and thriving. In this scenario, citizens have extremely limited control over their food practices. Their lifestyles become fully data-driven and manipulated in a top-down manner. Similar dystopian proposals for topdown health-diet data tracking exist in sci-fi literature [21], design fiction [8] and commercial food services [40]. At the workshop, the Total Food Control scenario provoked a debate on security aspects of diet tracking technologies. While quantified control over personal diets may help improve consumers' health, such data tracking risks privacy infringements from third parties - a nuance that must be taken seriously in HFI to support ethical diet tracking.

In contrast, a Future Food Roleplaying scenario created by another group, described a co-dining platform that connects distant diners to simulate shared dining experience and revive beloved food-related memories. The scenario is inspired by the Foodcasters card (describing remote codining practices) and an HFI project that experiments with digital tracking and revival of food-related memories via multi-sensory cues [1]. In the scenario, technology positively enhances social food interactions and promotes emotional wellbeing (Figure 6). Some participants argued against this techno-optimistic vision, noting that foodrelated memories as well as traditional cooking skills and dishes cannot be reproduced by technology with the complexity they deserve. The contextual, social and emotional sensitivities are simply too hard to capture.

To provide an example, a participant recounted her relationship to Pavlova - a tutu-shaped meringue-based dessert covered in cream and fruit, named after the ballerina Anna Pavlova. The dessert was a family favourite and her grandmother's signature dish. However, the grandmother consistently considered her Pavlova a failure. As a moneysaving strategy, she would use three eggs instead of six. As

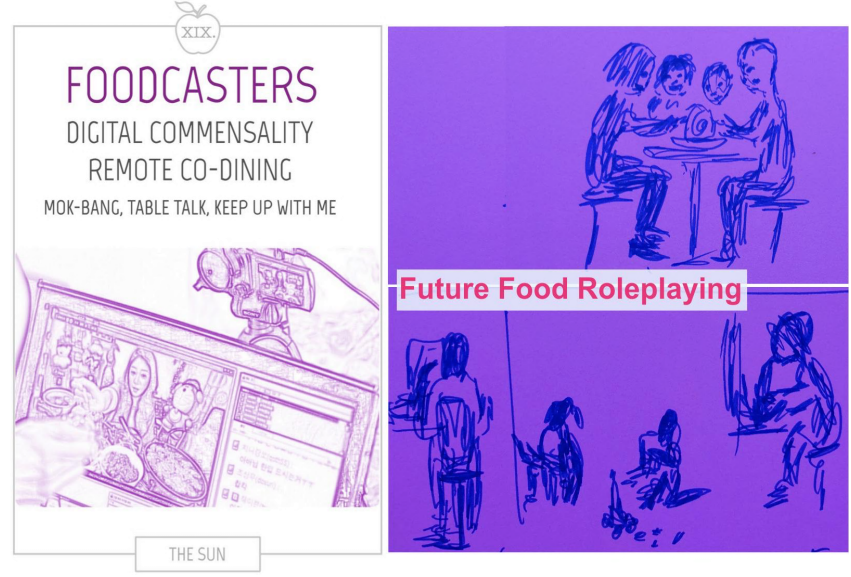

Figure 6: Future Food Roleplaying. To revive food-related memories, users are assigned to a dining group and assume a specific social role (e.g., in a family: mother, child, grandparent etc.) Gathered around a virtual dinner table, they re-enact food-related memories that might be hard to experience otherwise, e.g. a family Christmas feast from years ago.

a result, the Pavlova was flat and crisp, instead of full and soft. Nonetheless, all family members enjoyed it, and it was a source of much gentle ribbing amongst the family. No one else could make Pavlova in the same way, despite having the grandmother's recipe. The complex, personal embodied expertise of Pavlova-making could not be captured in text. The experience of eating the dessert made and served by anyone else was different: it lacked the crunchy texture of the grandmother's 'failed' version and the accompanying emotions. This account prompted a rich discussion about how traditional hand-made dishes are much more than a combination of ingredients. They are the products of tacit knowledge carrying distinct social and emotional meanings. A recipe is a script for the performance of making a dish: while the recipe is replicable and does not need to change in time and space, the performance is intrinsically contextual and always different. We determined that HFI designers and researchers should be mindful of such social and emotional sensitivities when designing technologies to capture and extend food experiences (for a more elaborate discussion, see [10]).

Both of these scenarios - Total Food Control and Future Food Roleplaying - extrapolate existing food-tech trends and associated issues into future visions. The Food Tarot deck affords this imaginative transposition. It prompts users to identify HFI issues and consider them in a speculative manner, unconstrained by existing socio-technical limits. By scaffolding speculation in this way, the cards - as an experimental food design tool - afford debate about desirable directions for future HFI research.

Key takeaways: HFI designs must be mindful of consumer privacy and allow consumers control over their personal data. Designers should approach food practices as complex, socially and emotionally charged events in everyday life, rather than technologically replicable variables. 


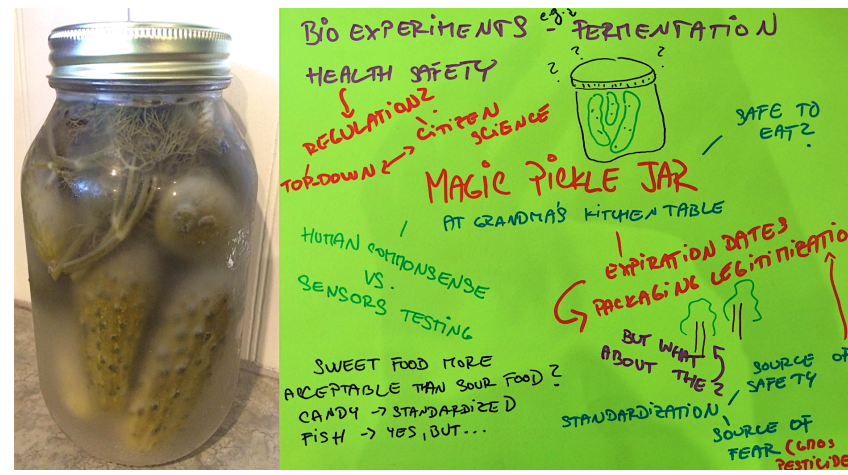

Figure 7: Pickle jar and discussions that emerged around.

\section{Reflecting through food-related boundary objects}

The DIS'19 workshop began with presentations of boundary objects that participants brought to represent a per sonal practice or perspective on HFI. These objects kickstarted our collective reflections. We describe three such objects and reflect on the critical perspectives they surfaced.

Grandmother's pickle jar. Form: photograph from grandmother's kitchen (Figure 7). Practice or perspective: differing models of food safety, trust, and responsibility.

Questions of trust often arise in relation to food, and can represent differing practices, perspectives, histories, and cultures. In the case of the grandmother's pickle jar, the participant considered the jar's contents potentially unsafe to eat, whereas her grandmother had no problem with the contents. The discrepancy arose because they use different criteria to assess food product safety. The participant is trained to prefer 'clean' food packaging with standardized expiration labels; her grandmother, a long-time fermentation practitioner, trusts her senses. The jar provoked a discussion about the contrast between 'new' food products or technologies aiming for 'clean' food practices and 'old' (traditional) food techniques - such as wild fermentation - that support 'messy' practices and experimental more-than-human food entanglements. While HFI designers must ensure the safety of their designs, they must also avoid eroding consumers' commonsense embodied, cultural, and historical - knowledge of food. At the workshop, we agreed that new food technologies should nurture curiosity for experimentation and commonsense practices of inspecting food, in preference to (presumed) safety through standardisation.

This recommendation aligns with the concern that consumers often misinterpret 'best-before' dates on food labels, to the detriment of responsible and sustainable food consumption [23]. It points to the societal benefit of empowering eaters to develop skills in food safety - using their senses to ascertain freshness. For example, smelling milk, testing floating eggs in water, and other historically common, scientifically validated practices.

At the workshop, we further considered how traditional

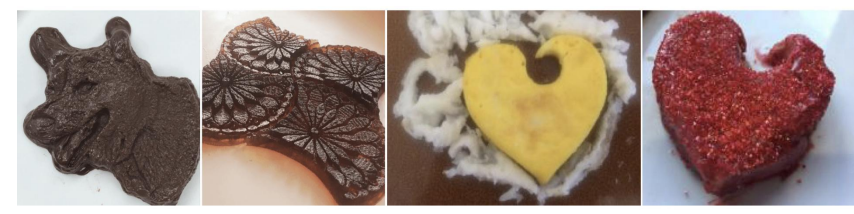

Figure 8: Experimental 3D printed food items - a melted chocolate, gelatin agar mix, egg yolk, and frozen popsicle.

food fermentation practices might assist us in developing beneficial relationships with our gut microbes - our visceral more-than-human companions. Making fermented foods (e.g., pickles, yogurt, kefir, beer) and caring for our ferments - 'feeding' them with sugar and starter cultures, avoiding cross-contamination - promotes their thriving. In return, consuming fermented food may nurture the diversity of our microbial flora and enable us to enjoy beneficial health effects [33]. Fermentation thus provides an occasion to learn about the importance of living organisms in our food systems; an approach often neglected in industrial food production, which tends to overlook the interdependencies of human and non-human life [2,15,35]. This notion is further attended to through our next boundary object:

DIY soil checker. Form: imagined (proposed) device. Practice or perspective: identifying relations between soil types and food [25].

More-than-human soil-food partnerships are essential to our food experiences: soil quality directly determines the quality of grown ingredients. This link is often invisible to end-consumers shopping for their groceries. For half a century, industrial agriculture has supported this 'invisibility', prioritizing yield and profit over soil health and food quality. Soil degradation is now recognized as a core factor in global climate and public health degradation [41]. The imagined DIY soil checker proposed to help unveil this invisibility. In response to this proposition, we discussed how urgent it is for food production strategies to reorient towards regenerative practices that prioritize morethan-human perspectives. We further recognised that it is critical for HFI authors to emphasize more-than-human concerns when designing food technologies. This expanded view - from Human-Food Interaction to 'More-thanHuman-Food Interaction' - allows for a more comprehensive consideration of the social and ecological consequences of food-tech innovations. We surmised that leveraging 'messy' traditional, experimental knowledge, such as that related to food fermentation, might assist designers and researchers in adopting and nurturing such comprehensive perspectives.

3D printed edibles. Form: 3D printed food items (Figure 8). Practice or perspective: digital fabrication to support human-food experimentation and creativity.

Novel fabrication techniques, such as 3D printing and laser cutting, open the door to intricate and (supposedly) accurate meal preparation. However, these techniques are culturally 
reductive, as they diminish traditional hands-on food practices and techniques. The participant who offered this boundary object specializes in digital food fabrication. Her experiments merge digital and traditional techniques to support creative food explorations [39]. Her sample 3D printed edibles (Figure 8) prompted thinking around the importance of critical framing of novel food technologies.

In commercial contexts, 3D food printing is often promoted as an extravagant technique to entertain aesthetic cravings through spectacular food shapes (that far outstrip the modest efforts of PancakeBot) or to solve problems 'through a click', without the need for conscious behaviour change. Examples of such proposals include printing with leftover food to avoid waste and printing with nutrient-rich materials to support healthy diets (overview: [31]). We discussed that while food printing technology may be a means to experiment with ingredients, textures, and shapes (as shown e.g. by the participant [39]) its ability to support playful hands-on learning about food materials and processes is currently underexplored. Majority of such technologies offer either a reductive solution or a mere spectacularity. Similar to the discussion held over our pancake experiments at DIS' 18 , we determined that humanfood interaction technologies should support creative food explorations and learning rather than quick-fix solutions accessible at the push of a button.

Key takeaways: HFI designs should encourage hands-on learning about food materials and nurture commonsense food knowledge, instead of prioritizing automation and standardization. Designers should leverage more-thanhuman perspectives to help pave the way towards environmentally sustainable food-tech innovation.

\section{Local food foraging \& tasting}

At two workshops, we undertook foraging excursions to frame our food-tech reflections in everyday-life contexts. At CHI'18, we walked around the conference venue, foraging for paper-based food waste to collectively craft a Food Tarot card with our bounty; at DIS'19, we organized an outdoor walk-shop to search for locally significant food items and dining experiences.

Food waste foraging in the $\mathrm{CHI}$ corridors was inspired by a 'locavore' version of the Food Tarot card deck. Before the workshop, a group of local participants mapped all 22 Food Tarot cards with local food venues (e.g. Gut Gardeners with a local fermentation workshop and Food NeoPunks with the Loop Juice shop that makes juice from leftover fruits [16]). From each of the 22 venues, they acquired paper-based waste, such as discarded food wrappings and used them as

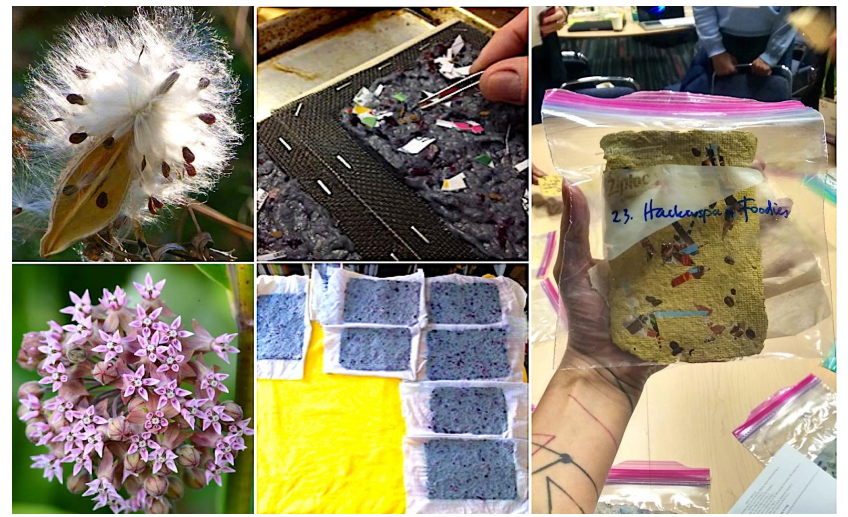

Figure 9: Collective crafting of a CHI Food Tarot card made of foraged food-related materials.

pulp for a 'local' Food Tarot deck. Each card was further embedded with milkweed seeds harvested in local wilderness (Figure 9). The tweaked local deck (set of Food Tarot cards made of montréalaise edible plants and trash) served as a reflective, trans-plantable food design artefact linking global food-tech trends with the local foodscape. At the workshop, all participants were invited to pick a card of their choice, take it home, plant it, and let it sprout thereby metaphorically sprouting and trans-planting the represented food-tech trends in their local food contexts.

Inspired by this reflective action, we collectively crafted a $\mathrm{CHI}$ food waste card from paper-based food waste gathered after conference coffee breaks and the milkweed seeds brought by the local participants (Figure 9). While making the card, we discussed how foraging - in the traditional sense of harvesting wild plants - can be an important sustainable food practice, but when implemented at scale may threaten local biodiversity. Human consumption of locally-foraged wild plants can leave less food for local insects and other non-human animals. It can impact soil, water, and other essential actants in the environment where the foraging takes place. A responsible foraging practice requires an intimate knowledge of a local ecosystem. Many food-tech foraging services, such as smartphone apps and crowdsourced maps [33], do not require users to have such local knowledge. Their use may thus exacerbate the negative potential of human foraging. Despite the intended goal of supporting sustainable practices, for instance by reducing supermarket shopping, such technologies may create more problems than they solve. This observation again steered our discussion towards the need to recognise more-than-human perspectives when designing for food practices and futures. 


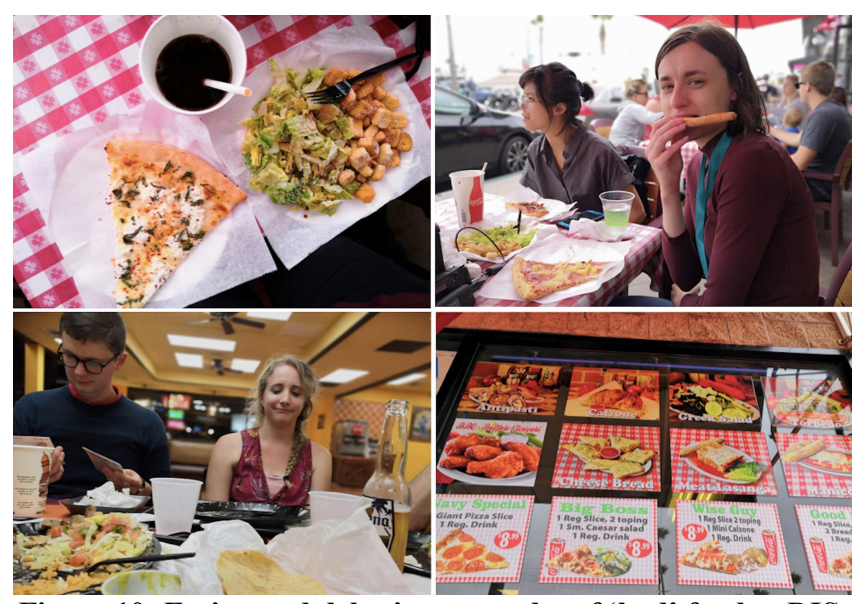

Figure 10: Eating and debating examples of 'bad' food at DIS.

The DIS'19 walk-shop was held around the San Diego SeaWorld resort (the conference venue). This location provided a controversial context for exploring food culture. Dining options consisted of expensive hotel restaurants, fast-food chains, and pizza parlours. We acknowledged our privilege of having 'the luxury of choice' in this extreme foodscape and took lunch in a local pizza joint. While eating pizza and sipping soda from gigantic plastic cups, we talked about unequal socio-economic access to 'good' (healthy, sustainable) food products and technologies designed to support 'good' food practices (Figure 10). Unhealthy and unsustainable diets are commonly associated with lower incomes [19,29]. Similarly, food-tech products designed to support better food lifestyles are often available only to people from privileged socio-economic backgrounds. Non-access due to income, education, or illiteracy can marginalise entire social groups. While feasting on our plastic-pizza lunch, we discussed the importance of HFI technologies being accessible to diverse socio-economic publics. If we are to avoid exacerbating existing, or creating new, inequalities on the global food market, we need to pay attention to existing socio-economic sensitivities.

During the walk-shop, we also foraged for local - found or purchased - boundary objects to capture the unequal availability of 'good' and 'bad' food in the area: a bag of pistachio nuts in a local bodega, considered a 'good' healthy snack, was far more expensive than other items on the snack shelf, such as fried crisps. On a nearby beachfront, a stand selling popsicles was the only available food option. We acquired a testing sample and, for lack of any 'good' options, constructed a 'DIY salad' of seaweeds and flowers foraged from the beach (Figure 11). These food items, together with written notes and photo documentation of the walk-shop, served as material for our final afternoon workshop session, where we collectively mapped food-tech issues and crafted an HFI Zine (described below).

The two foraging walk-shops illustrate the situated nature of human-food practices. They reminded us that such practices cannot be adequately interpreted from a research

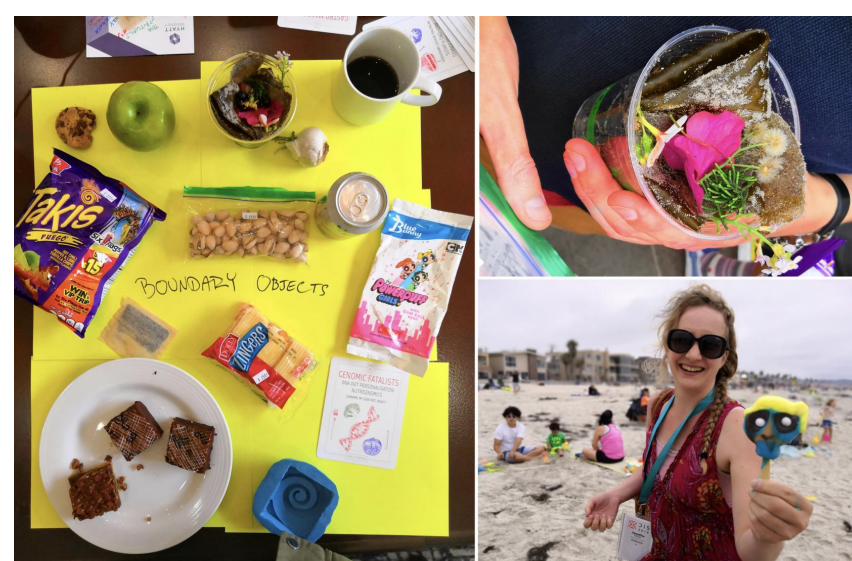

Figure 11: Foraged 'good' and 'bad' food boundary objects.

lab or design studio. Rather, they must be explored through fieldwork that situates the 'research lab in the wild' [36]. HFI would benefit from embracing such performative locally-aware research approaches, which provide an opportunity to better grasp local sensitivities of food practices, and aid design.

Key takeaways: HFI designs should enable equitable access and aim to reduce inequalities on the global food market rather than expand them. 'Research labs in the wild' can enable designers to carefully reflect contextual sensitivities and situated nature of local food cultures and practices.

\section{Mapping HFI landscape \& distilling recommendations} At DIS'19, we used our HFI Lit Review $A^{1} p^{1}$ - a community-driven online database of published HFI research [3]. The aim was to facilitate a focused discussion about the HFI landscape and reach beyond research projects presented by workshop participants. We used the 'app' to search for examples of HFI projects addressing issues raised during our previous co-creation activities. This exercise helped us to identify opportunities for future research projects and collaborations, as well as gaps in the existing corpus of HFI research. Reflecting on the previously discussed need to emphasize more-than-human perspectives in HFI, we searched for available texts addressing this topic. At the time, there was little related HFI research. Regenerative agriculture, soil-food interaction, and other topics related to 'More-than-HumanFood Interaction' were not well represented in the research literature. Our first move in response to this gap, is a DIS'20 workshop [15].

To conclude the DIS'19 workshop, we initiated a collaborative HFI Zine, summarising our discussions, debates, scenarios, and walk-shop activities. We shared our walk-shop documentation - photos, drawings, notes, sampled boundary objects - and mapped them on a large canvas. We collectively distilled the core points, represented them in graphical form, and turned them into the first Zine pages (Figure 12). A zine is a feasible

\footnotetext{
${ }^{1}$ The app can be accessed at: https://www2.ucsc.edu/hfi/
} 


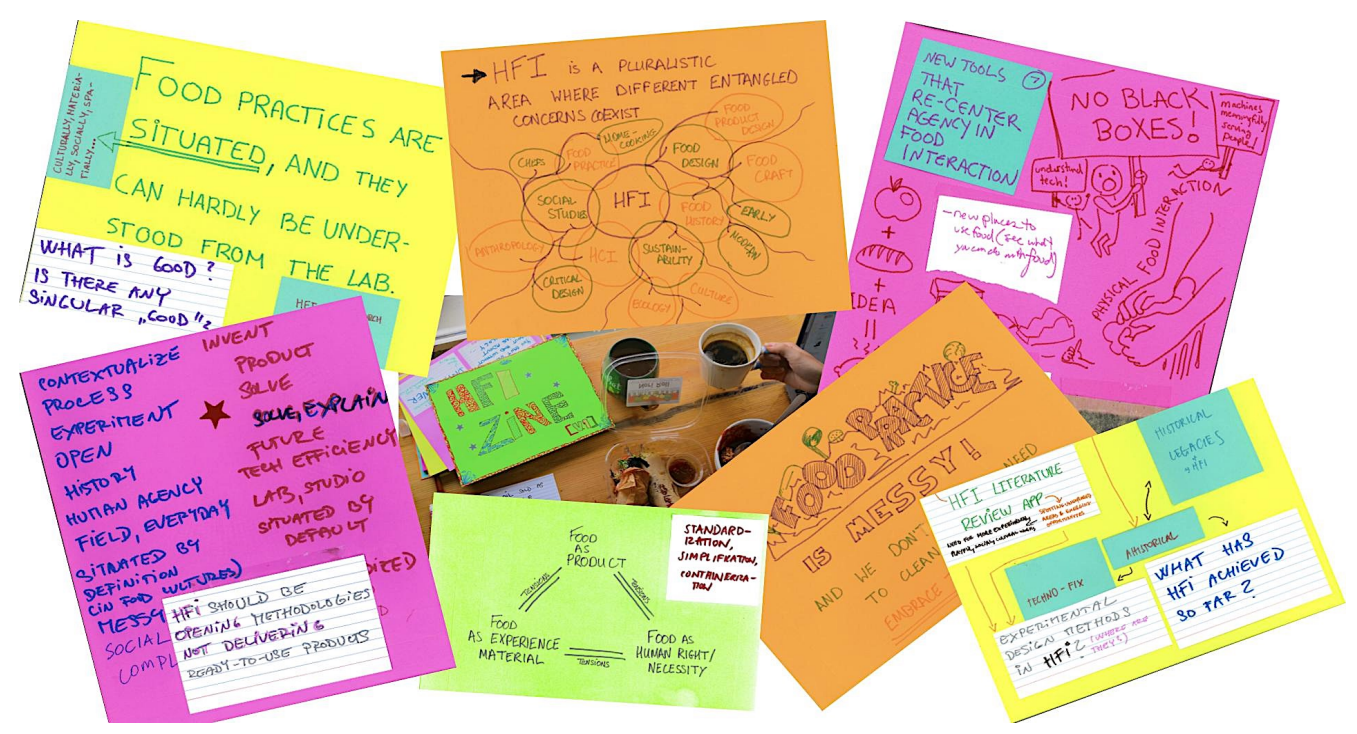

Figure 12: HFI Zine collectively initiated at DIS'19 workshop.

medium to organize thoughts-in-progress in a visually appealing way and disseminate them beyond the workshop, in an open-access format, designed to reach diverse audiences. The HFI Zine was later extended with findings from all FFF workshops and self-published online [18].

The finalised Zine serves as a condensed summary of HFI opportunities, issues, proposals, imaginaries and desirable future directions of the field. Not a manifesto or fixed set of guidelines, but rather a humble set of ideas, the Zine offers a suite of recommendations, bringing together the key takeaways from each workshop (Table 1).

\section{COMPOSTING TANGIBLE DESIGN}

In each of the experiments described, we used food and eating as method, medium, and context for research. We made use of foodstuffs, food packaging, food-based props, food techniques, practices, cultures and traditions. We took into account the organoleptic - tangible, sensually stimulating - qualities of food, its accessibility, and its varied meanings, as understood through the first-person perspectives and co-creative activities of our workshop participants. Through this rich materiality, we engaged with food as a research subject. Throughout our experiments, the 'liveness' of food, its sensorial richness and edibility, is inescapable. This same trait is what makes food perishable and compostable. We find that these attributes are largely overlooked in design when considering materials for experimental use. Design research uses tangible materials as a core element, not only when developing artefacts, but also during experimentation (see [30] for a raft of examples). Many of the materials used are unsustainable, or certainly less sustainable than food. Through our food research, we engage deeply with the tangible, perishable, compostable, and comestible characteristics of food. Our material decomposes both during and after our experiments. Rather than see these traits as a problem, we consider them step towards that consideration, we have begun to frame our choice of materials through the question: what if it was edible? When answered in the affirmative, we are moving a

To support creative, equitable, and sustainable more-than-human food practices, HFI should:

- Open spaces for creative food experimentation, attending to the full organoleptic experience of food, rather than deliver quick-fix solutions aimed at consumer convenience.

- Nurture food experiences that are socially engaging, culturally aware, and playful by taking inspiration from diverse local food knowledges and traditions.

- Be mindful of consumer privacy and keep consumers in control over their personal data.

- Approach food practices as complex, socially and emotionally charged events in everyday life rather than technologically replicable variables.

- Encourage hands-on learning about food materials and nurture commonsense food knowledge instead of prioritizing automation and standardization.

- Enable equitable access and help reduce inequalities on the global food market rather than expand them.

- Undertake 'research labs in the wild' to carefully reflect contextual sensitivities and the situated nature of local food cultures and practices.

- Leverage more-than-human perspectives to support environmentally sustainable food-tech innovation.

- Shift from designing for Human-Food Interaction to designing for More-than-Human-Food Interaction.

Table 1: Key recommendations for HFI. 
step closer to ecosystem integration and regenerative, experimental practices that position design as a positive contributor, not only to our social environs but to our vibrant, multi-species ecosystem.

\section{DIGESTING OUR FINDINGS}

In describing our four workshops, we demonstrate a variety of ways that experimental food design co-creation can support collective sense-making in HFI. We presented six distinct experimental design approaches tested at the workshops. These approaches make use of food as both design material and starting point for reflection. They are playful, imaginative, and use food (in its many instantiations) to provoke embodied, creative social interactions and critical reflections among diverse stakeholders. Our experiments demonstrate how foodrelated 1) crafting, 2) situated food-play design, 3) speculating, 4) boundary objects, 5) foraging, and 6) mapping can be leveraged to nurture critical debates on important HFI issues. Drawing on the debates at the workshops and the design materials produced collaboratively with the participants, we proposed a humble set of recommendations for creative, sustainable, socially and ecologically just HFI design and research.

Our workshops bring focus to the ambivalent impact that new food technologies can have on food cultures. Foodtech products and services designed to improve practices and solve problems often create problems of their own. Technologies can reduce socio-culturally and sensoriallyrich food experiences into utilitarian, standardized tasks performed by algorithms. They can erode the playful and creative potential embedded in material food practices, compromise consumer privacy, and bring other challenges to the table. However, this is not to say that we should strive for non-tech food futures. With increasing environmental instabil ity, food security issues and public health crises, it is becoming urgently evident that food practices must change. Indeed, the way we eat and provision and dispose of our food at present is pressuring earth system tipping points and contributes to all seventeen of the UN World Sustainability Development Goals (SDGs), not always in positive ways [38]. Human-food practices are making both people and the planetary system on which we rely for our survival, sick. We believe technology innovation can be a viable approach to facilitate transformational changes in the food sector, whether these changes are systemic or experienced in simple, everyday practices as we sit around the table. To incorporate technology meaningfully into our food experiences, foodtech designers and researchers must be mindful to carefully consider the diverse impacts that innovation may have on food cultures, and embrace a more-than-human perspective to avoid unnecessary risk and harm.

\section{CONCLUSION}

The debates and recommendations we highlight here reflect important issues that we believe HFI authors should consider as food-tech innovation moves forward. While provoked by the workshop activities (categorized here into six approaches), these debates illustrate how experimental food design co-creation can serve to support critical engagement of diverse stakeholders with urgent sociotechnical issues - such as those emerging in food systems. Creative, experimental approaches in art and design research provide a promising way to initiate and extend engagements with emerging social issues and inspire collective reflection about how things might be different [24]. Our workshops illustrate the viability of such approaches in the specific context of food. However, we also see potential in using food-related experimental design to support critical stakeholder engagements with other than food-tech issues. Food and eating are relatable everydaylife materials and events. They can serve as culturally rich, personally, politically and environmentally potent starting points from which to provoke critical hands-on engagements with a range of themes and issues.

Beyond the relevance and familiarity of food as subject and material, the edibility, perishability and thus compostability of food are key attributes that can be leveraged by designers who use tangibles in design research. Tangibles are used for many purposes, for instance, in interviews and workshops to (co-)construct knowledge and understanding $[9,30]$. Unless tangibles are required to last 10,000 years (e.g. for an interstellar voyage), we suggest designers consider the perishability and compostability of the materials they use to fabricate their tangibles, in addition to commonly considered qualities such as rigidity, flexibility and transparence. If sustainability is taken seriously, and responsibility seen to extend beyond the recycle bin, designers may begin to appreciate materials with shorter lifespans, and whose qualities evolve over time. Doing so may enliven design research processes as the material artefacts evolve somewhat outside of the control of the designer. While our research in this area is fledgling $[12,13,35]$, we see it as a gap in design research that warrants attention. With this in mind, we hope that our observations and remarks inspire also designers and researchers working outside the HFI domain who have an interest in designing creative socio-culturally sustainable experiences. To conclude, we recognise a continuity in the conversations we initiated within the workshops, and acknowledge the importance of increasing the diversity of stakeholders at the table to include a broader set of perspectives on food-tech and HFI. The Feeding Food Futures (FFF) network [17] pursues this goal and aims to encompass a broad spectrum of food-oriented researchers, designers, practitioners, and (human and nonhuman) eaters of diverse backgrounds.

\section{Acknowledgements}

We thank our workshop co-authors and participants, past and future, who are key to shaping the activities and discussions we organize around HFI. 


\section{REFERENCES}

[1] Passant El Agroudy. 2018. What Does My "Comfort Food" Say About My Memories? Presented at 2018 CHI Conference Workshop on Designing Recipes for Digital Food Futures. Retrieved January 10, 2020 from https://bit.ly/34WPfpr

[2] Algorithmic Food Justice. 2020. Retrieved January 10, 2020 from http://algorithmicfoodjustice.net

[3] Ferran Altarriba Bertran, Samvid Jhaveri, Rosa Lutz, Katherine Isbister, and Danielle Wilde. 2019. Making Sense of Human-Food Interaction. In CHI Conference on Human Factors in Computing Systems Proceedings (CHI'19). https://doi.org/10.1145/3290605.3300908

[4] Ferran Altarriba Bertran, Elena Márquez Segura, Jared Duval, and Katherine Isbister. 2019. Chasing Play Potentials: Towards an Increasingly Situated and Emergent Approach to Everyday Play Design. In Proceedings of the ACM Conference on Designing Interactive Systems (DIS'19). https://doi.org/10.1145/1858171.1858228

[5] Ferran Altarriba Bertran, Danielle Wilde, Ernő Berezvay, and Katherine Isbister. 2019. Playful Human-Food Interaction Research: State of the Art and Future Directions. In Proceedings of the 2019 Annual Symposium on Computer-Human Interaction in Play (CHI Play‘19). https://doi.org/10.1145/3322276.3322325

[6] Ferran Altarriba Bertran, Danielle Wilde, Elena Márquez Segura, Oscar Garcia Pañella, Laia Badal León, Jared Duval, and Katherine Isbister. 2019. Chasing Play Potentials in Food Culture to Inspire Technology Design. In Proceedings of the 2019 Annual Symposium on Computer-Human Interaction in Play (CHI Play'19 EA).

https://doi.org/10.1145/3341215.3349586

[7] Lloyd Alter. 2016. Is the PancakeBot the best thing ever or just another piece of electronic crêpe? Retrieved April 22, 2020 from https://bit.ly/3asI31Z

[8] Burton-Nitta. 2011. Republic of Salivation. Retrieved January 10, 2020 from https://bit.ly/2RYc0UI

[9] Jacob Buur and Ben Matthews. 2008. Participatory innovation. International Journal of Innovation Management 12, 03, 255-273.

[10] Hilary Davis, Bjorn Nansen, Frank Vetere, Toni Robertson, Margot Brereton, Jeannette Durick, and Kate Vaisutis. 2014. Homemade cookbooks: a recipe for sharing. In Proceedings of the 2014 conference on Designing interactive systems (DIS'14). http://dx.doi.org/10.1145/2598510.2598590.

[11] Markéta Dolejšová, Ferran Altarriba Bertran,Danielle Wilde, and Hilary Davis, 2019. Crafting and Tasting Issues in Everyday Human-Food Interactions. In 2019 ACM Conference Companion Publication on
Designing Interactive Systems (DIS'19).

https://doi.org/10.1145/3301019.3319994

[12] Markéta Dolejšová and Denisa Kera. 2016. The Fermentation GutHub Project and the Internet of Microbes. Enriching Urban Spaces with Ambient Computing, the Internet of Things, and Smart City Design. IGI Global.

[13] Markéta Dolejšová, Tereza Lišková, and Martin Obert. 2017. HotKarot \& OpenSauce: Edible Storytelling \& Design Speculations. Proceedings of the 3rd Biennal Conference on Research Through Design (RTD'17). https://doi.org/10.6084/m9.figshare.4746982.v1

[14] Markéta Dolejšová, Rohit Ashok Khot, Hilary Davis, Hasan Shahid Ferdous, and Andrew Quitmeyer. 2018. Designing Recipes for Digital Food Futures. In 2018 Conference on Human Factors in Computing Systems (CHI EA'18). https://doi.org/10.1145/3170427.3170622

[15] Markéta Dolejšová, Sjef van Gaalen, Danielle Wilde, Paul G. Raven, Sara Heitlinger, and Ann Light. 2020. Designing with More-than-Human Food Practices for Climate-Resilience. Workshop accepted for DIS 2020.

[16] Natalie Doonan, Pamela Tudge, and David Szanto. 2018. Digital Food Cards: YUL Forage. Presented at 2018 CHI Conference Workshop on Designing Recipes for Digital Food Futures. Retrieved January 10, 2020 from https://bit.ly/2XUOewB

[17] Feeding Food Futures. Retrieved January 10, 2020 from http://foodfutures.group

[18] Feeding Food Futures. 2019. HFI Zine. Retrieved January 10, 2020 from http://bit.ly/HFIzine

[19] Simone A. French, Christy C. Tangney, Melissa M. Crane, Yamin Wang, and Bradley M. Appelhans. 2019. Nutrition quality of food purchases varies by household income: the SHoPPER study. BMC public health 19, 1, 231.

[20] Julian Hanna. 2019. An overview of contemporary speculative practice. SpeculativeEDU. Retrieved January 10, 2020 from https://speculativeedu.eu/anoverview-of-contemporary-speculative-practice/

[21] Aldous Huxley. 2007. Brave new world. Ernst Klett Sprachen.

[22] June. Retrieved January 10, 2020 from https://juneoven.com/

[23] Sheena Leek, Isabelle Szmigin, and Emily Baker. 2015. Consumer confusion and front of pack (FoP) nutritional labels. Journal of Customer Behaviour 14, 1, 49-61.

[24] Ann Light, Ruth Wolstenholme, and Ben Twist. 2019. Creative practice and transformations to sustainabilityinsights from research. SSRP Working Paper No. 
2019-1, University of Sussex. Retrieved January 10, 2020 from https://bit.ly/2RYLYjS

[25] Elena Márquez Segura, Laia Turmo Vidal, Asreen Rostami, and Annika Waern. 2016. Embodied Sketching. In Proceedings of the 2016 CHI Conference on Human Factors in Computing Systems (CHI'16). https://doi.org/10.1145/2858036.2858486

[26] Huiying Ng. 2019. Recognising the edible urban commons: Cultivating latent capacities for transformative governance in Singapore. Urban Studies 1-17. https://doi.org/10.1177/0042098019834248

[27] Homin Park, Abhinav Ramesh Kashyap, Zhenkai Wang, and Brian Y. Lim. 2018. Biases in Food Photo Taking Behavior. Presented at CHI 2018 Workshop: Designing Recipes for Digital Food Futures. Retrieved January 10, 2020 from https://bit.ly/2VvcXWv

[28] Parlour of Food Futures. Retrieved January 10, 2020 from https://foodtarot.tech/

[29] Kathryn L Clark, R Vincent Pohl, and Ryan C Thomas. 2019. Minimum Wages and Healthy Diet. Contemporary Economic Policy.

[30] Elizabeth B-N Sanders and Pieter Jan Stappers. 2012. Convivial toolbox: generative research for the front end of design. Bis Publisher.

[31] Bethaney Turner and Deborah Lupton. 2017. 'Both fascinating and disturbing': Consumer responses to 3D food printing and implications for food activism. In Digital Food Activism, Routledge, 169-185.

[32] Erica Vannucci, Ferran Altarriba, Justin Marshall, and Danielle Wilde. 2018. Handmaking Food Ideals: Crafting the Design of Future Food-related Technologies. In 2018 ACM Conference Companion Publication on Designing Interactive Systems (DIS'18). https://doi.org/10.1145/3197391.3197403
[33] Baohong Wang, Mingfei Yao, Longxian Lv, Zongxin Ling, and Lanjuan Li. 2017. The human microbiota in health and disease. Engineering 3, 1, 71-82.

[34] Wild Edibles Forage. Retrieved January 10, 2020 from https://apple.co/2xSEIzf

[35] Danielle Wilde. 2019. Tasting the Future. Anticipation 2019, AHO, Oslo, Oct. 2019. Retrieved January 10, 2020 from https://bit.ly/376LygK

[36] Danielle Wilde, Jenny Underwood, and Rebecca Pohlner. 2014. PKI: crafting critical design. In Proceedings of the 2014 conference on Designing Interactive Systems (DIS'14).

[37] Danielle Wilde, Anna Vallgårda, and Oscar Tomico. 2017. Embodied design ideation methods: analysing the power of estrangement. In Proceedings of the 2017 CHI Conference on Human Factors in Computing Systems, (CHI'17).

[38] Walter Willett et al. 2019. Food in the Anthropocene: the EAT-Lancet Commission on healthy diets from sustainable food systems. The Lancet 393, 10170, 447492.

[39] Jennifer Weiler. 2017. 3D Print-Based Silicone Mold Culinary Study. SANDS. Retrieved January 10, 2020 from https://sandsystems.org/2017/08/19/3d-printbased-silicone-mold-culinary-study/

[40] Zipongo. Retrieved January 10, 2020 from https://meetzipongo.com/

[41] Lu Zhang, Chengxi Yan, Qing Guo, Junbiao Zhang, and Jorge Ruiz-Menjivar. 2018. The impact of agricultural chemical inputs on environment: global evidence from informetrics analysis and visualization. International Journal of low-Carbon technologies 13, 4, 338-352. 